\title{
X-Ray Scattering from Freestanding Polymer Films with Geometrically Curved Surfaces
}

\author{
D. R. Lee, ${ }^{1}$ K. Shin, ${ }^{2}$ O. H. Seeck, ${ }^{3}$ Hyunjung Kim, ${ }^{4}$ Y.-S. Seo,${ }^{5}$ M. Tolan, ${ }^{6}$ M. H. Rafailovich, ${ }^{5}$ \\ J. Sokolov, ${ }^{5}$ and S. K. Sinha ${ }^{7}$ \\ ${ }^{1}$ Advanced Photon Source, Argonne National Laboratory, Argonne, Illinois 60439, USA \\ ${ }^{2}$ Department of Material Science and Engineering, Kwangju Institute of Science and Technology, Kwangju 500-712, Korea \\ ${ }^{3}$ IF F, FZ Jülich GmbH, 52425 Jülich, Germany \\ ${ }^{4}$ Department of Physics, Sogang University, Seoul 121-742, Korea \\ ${ }^{5}$ Department of Materials Science and Engineering, State University of New York, Stony Brook, New York 11794, USA \\ ${ }^{6}$ University of Dortmund, Experimentelle Physik I, D-44221 Dortmund, Germany \\ ${ }^{7}$ Department of Physics, University of California, San Diego, La Jolla, California 92093, USA, \\ and Los Alamos National Laboratory, Los Alamos, New Mexico 87545, USA
}

(Received 30 November 2002; published 8 May 2003)

\begin{abstract}
We show that the $\mathrm{x}$-ray surface scattering from a freestanding polymer film exhibits features that cannot be explained by the usual stochastic formalism for surfaces with random height fluctuations. Instead, a geometric description of the film morphology assuming two curved surfaces characterized by a radius of curvature and a lateral cutoff length successfully accounts for the phase difference between the Kiessig fringes of the nominal "specular" and "off-specular" components of the scattering. The formalism allows one to distinguish unambiguously between conformal and anticonformal curvature morphologies at long length scales.
\end{abstract}

DOI: 10.1103/PhysRevLett.90.185503

PACS numbers: 61.10.Kw, 68.47.Mn, 68.55.Jk

While the formalism for discussing the x-ray scattering from curved surfaces has been known for some time [1], all specular and off-specular x-ray reflectivity experiments both supported and freestanding on thin films have been interpreted in terms of correlation functions involving a statistical description of random height fluctuations on surfaces which are on the average flat and parallel. For films where the height fluctuations are uncorrelated, Kiessig fringes (i.e., oscillations in the scattering intensity as a function of $q_{z}$, the wave vector transfer normal to the film surface) appear in the specular reflectivity but not in the off-specular scattering. If conformally correlated height fluctuations exist, as shown in Fig. 1(a), Kiessig fringes appear in both the specular and off-specular scattering and are in phase [2-4]. If islands of constant height exist on the surface, the Kiessig fringes are exactly out of phase [4,5]. During the past decade, $\mathrm{x}$-ray specular and off-specular reflectivities have been successfully employed to disclose a degree of conformality between different interfaces in many thin film systems, such as metallic multilayers [2,6,7], LangmuirBlodgett films [8], and smectic liquid crystal films [9-11].

In this Letter, we present an example of a system where such a statistical description fails, and instead a geometric description of the film surface morphology in terms of surfaces curved at long length scales is necessary to describe the scattering function. The system consists of a freestanding polymer film with a dilute surface concentration of lens-shaped asperities or "bubbles" with a mean separation which is greater than the coherence length of the $\mathrm{x}$ rays on the film surface. It is shown that the scattering exhibits a fringe pattern which can be used to determine the average lateral size and radius of curva-

ture of the lens-shaped regions with great accuracy. This approach can unambiguously distinguish between $\mathrm{x}$-ray scattering from conformal [Fig. 1(a)] and anticonformal [Fig. 1(b)] surface morphologies on the freestanding film surfaces. Also, this technique provides an almost unique tool to obtain information on the correlations between the morphologies of two curved surfaces, which cannot be obtained from other surface-sensitive techniques, for example, atomic force microscopy, to our knowledge.

For a demonstration, $\mathrm{x}$-ray reflectivities were measured from a very thin freestanding polystyrene film with a molecular weight $M_{w}=650 \times 10^{3}$ and a polymer radius of gyration $R_{G} \sim 220 \AA$ [12]. The x-ray reflectivity measurements were performed at beam line X10B of the National Synchrotron Light Source using a monochromatic beam of wavelength $\lambda=1.127 \AA$. Figure 2 illustrates schematically the geometry of surface scattering, with directions in reciprocal space superimposed on the real space diagram. $\mathbf{q}$ represents the wave vector transfer $\left(\mathbf{k}_{f}-\mathbf{k}_{i}\right)$. Various directions in $\mathbf{q}$ space can be scanned by appropriate settings of the incidence and exit angles $\theta_{i}$ and $\theta_{f}$ or, equivalently, the sample tilt and the detector angle. $q_{x}$ and $q_{y}$ are the components of $\mathbf{q}$ in the plane of the film. Thus, one may scan from the origin of $\mathbf{q}$ space out along $q_{z}$ (true specular with $\theta_{i}=\theta_{f}$ ) or in directions transverse to the specular, i.e., along $q_{x}$ ("transverse"

(a)

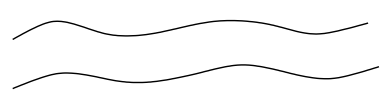

FIG. 1. Schematic representation of conformal (a) and anticonformal (b) surface morphologies on thin films. 


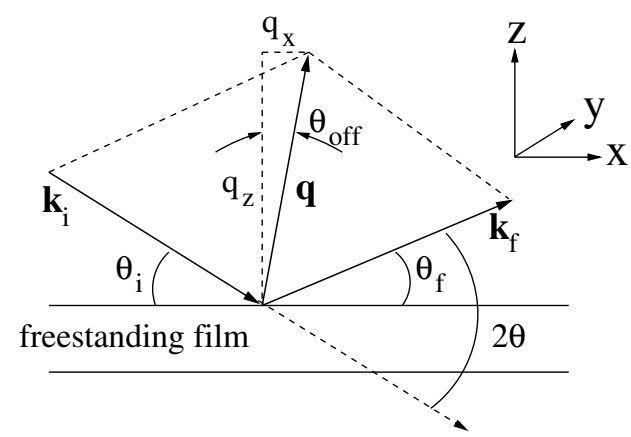

FIG. 2. The in-plane scattering geometry. Specular scan is along $q_{z}$, longitudinal diffuse scans are along the vector $\mathbf{q}$ as shown, and transverse scans are primarily along $q_{x}$ as indicated.

scans), by means of so-called "rocking curves" [tilting the sample in the beam with the total scattering angle $2 \theta=\left(\theta_{i}+\theta_{f}\right)$ fixed] or along off-specular directions making an offset angle $\theta_{\text {off }}=\left(\theta_{i}-\theta_{f}\right) / 2$ to $q_{z}$ ("longitudinal" scans). For small angles, the resolution widths of in-plane scattering are given by $\Delta q_{z}=1.32 \times 10^{-3} \AA$ and $\Delta q_{x}=1.18 \times 10^{-4} q_{z}$. The slits were set wide in the direction normal to the scattering plane shown in Fig. 2, leading to an effective integration of the scattering over $q_{y}$.

Figure 3 shows the results of specular $\left(\theta_{\text {off }}=0^{\circ}\right)$ and longitudinal off-specular $\left(\theta_{\text {off }}=-0.1^{\circ}\right.$ and $\left.+0.1^{\circ}\right)$ reflectivity measurements from the sample. Specular reflectivity intensities in Fig. 3(a) show the Kiessig fringes corresponding to the film thickness of $314.8 \AA$. Two off-specular scan intensities also show the interference fringes clearly, indicating strong vertical correlation between the two interfaces. However, among these three curves, the positions of maxima and minima in the interference fringes are shifted relative to each other along the $q_{z}$ direction, as indicated by the vertical dashed lines in Fig. 3, unlike the case of conformal fluctuations where the oscillations in the specular and off-specular scans are in phase. It has also been observed that the longitudinal off-specular reflectivity has an oscillation exactly out of phase with those in the specular reflectivity from a surface decorated with islands of constant height, which has been explained by assuming a bimodal distribution for the height function $[4,5]$.

The phase relationship between the fringes in the specular and off-specular scans observed here changes as a function of $q_{z}$ and is thus more complicated than in the above cases. The transverse off-specular scans in Fig. 4 show a sharp peak, which represents the specularly reflected beam from the smooth part of the film superimposed on a broader peak arising from the lenses. This broader intensity distribution alternates as a function of $q_{z}$ between having a single maximum in the center to having a minimum in the center surrounded by maxima on either side, and the interference fringe pattern that we have studied in the off-specular direction appears only

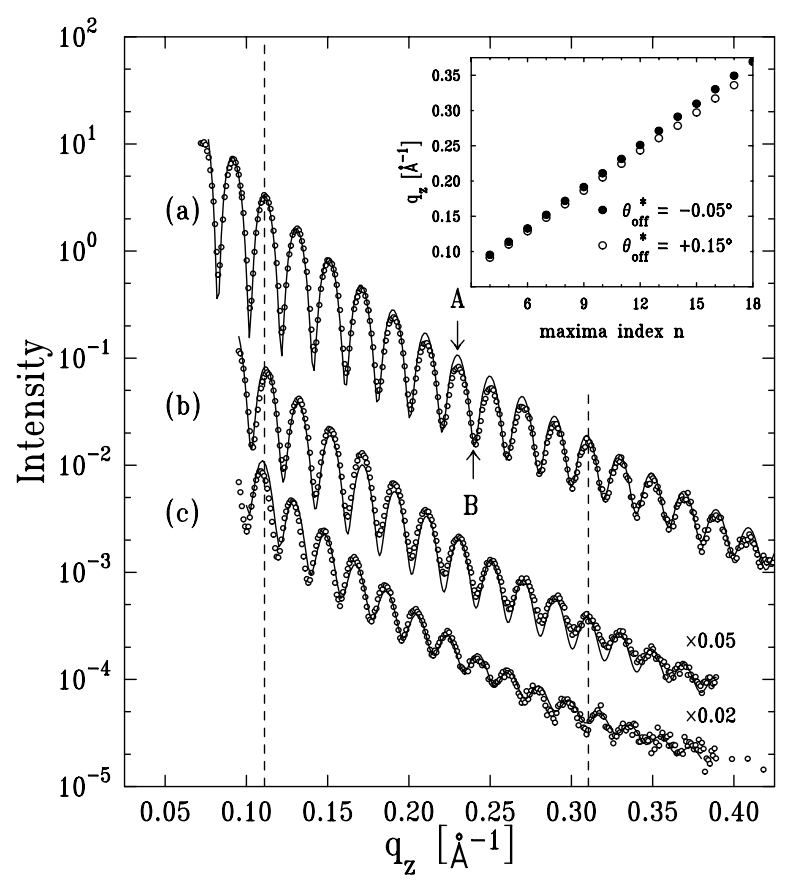

FIG. 3. X-ray specular (a) and longitudinal off-specular reflectivity scans with offset angles of $-0.1^{\circ}$ (b) and $+0.1^{\circ}$ (c) from a freestanding polystyrene thin film with the average thickness of $314.8 \AA$. The circles are data, and the lines are fits. For clarity, the two off-specular intensities are scaled to not overlap. The vertical dashed lines are guides to the eye to compare the phase in maxima positions of the oscillations among different types of reflectivity curves. Arrows indicate the values of $q_{z}$, where transverse rocking scans have been performed as shown in Fig. 4. The inset shows the $q_{z}$ values of maxima of the oscillations in off-specular reflectivities as a function of index $n$. Note that nominal offset angles, $-0.1^{\circ}$ and $+0.1^{\circ}$, correspond effectively to $-0.05^{\circ}$ and $+0.15^{\circ}$, respectively, with respect to the center of the broad peak in Fig. 4.

in this broad distribution. We now construct a model surface morphology which can explain the above feature.

Let us consider a film with a random and sparse distribution of lens-shaped islands on an otherwise flat background where the film surfaces are parallel [Fig. 5(a)]. The latter will yield the resolution-limited specular peak. We assume that because of the sparseness and randomness of the lenses, the off-specular scattering can be written as the sum of the normal capillary wave scattering from the flat regions alone and the incoherent superposition of the scattering from each of the lens-shaped regions. We further assume that the lateral size of each lens-shaped island is smaller than the projected coherence length of the $\mathrm{x}$ rays along the surface but that the spacing between lenses is larger than this coherence length, justifying the neglect of the interference between scattering from different islands. Finally, it should be pointed out that there is an offset of $-0.05^{\circ}$ between the centers of two components, the specular peak and the broader off-specular one, as shown in Fig. 4, indicating a tilt of the equatorial plane of the lenses with respect to the average surface of the rest of 


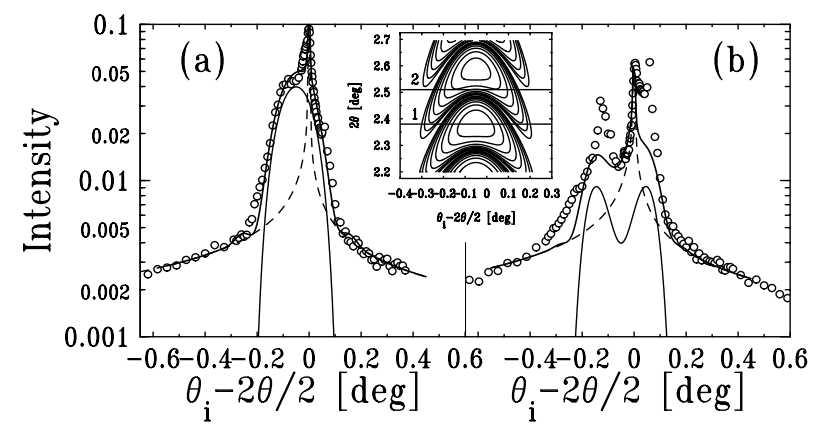

FIG. 4. Transverse rocking scans at the crest (a) and the valley (b) of the oscillations in specular reflectivity, indicated by arrows $A$ and $B$ in Fig. 3, respectively. The thick solid lines represent fits. The thin solid lines and dashed lines represent the contributions from curved surfaces and capillary wave fluctuations, respectively. The intensities are shown as a function of $\left[\theta_{i}-(2 \theta / 2)\right]$, while $q_{x}=q_{z} \times \sin \left[\theta_{i}-(2 \theta / 2)\right]$. The inset shows the intensity distribution from curved surfaces using Eq. (4). The horizontal lines 1 and 2 represent the trajectories of the rocking scans at the crest (a) and the valley (b), respectively.

the film. Since these freestanding films are viscoelastic rather than liquid when their thicknesses are on the order of $R_{G}$ [12], there can exist macroscopic strains in the film arising from the edges where it is supported which may account for this phenomenon. It is, however, not the focus of the present work, and thus we analyze the scattering from the lenses by shifting the origin to the tilted value of theta.

To consider the scattering from a single lens-shaped island, we choose the $(x, y)$ plane as the average plane for this island. Following Ref. [1], the differential cross section for scattering from a freestanding film with density $\rho_{0}$ can be given in the kinematical approximation by

$$
\begin{aligned}
\frac{d \sigma}{d \Omega}=\frac{\rho_{0}^{2}}{q_{z}^{2}} \mid & \iint d x d y e^{-i q_{z} z_{2}(x, y)} e^{-i\left(q_{x} x+q_{y} y\right)} \\
& -\left.\iint d x d y e^{-i q_{z} z_{1}(x, y)} e^{-i\left(q_{x} x+q_{y} y\right)}\right|^{2},
\end{aligned}
$$

where $z_{1(2)}(x, y)$ is the height of the air/film (film/air) surface at lateral position $(x, y)$. We should mention here that the kinematic approach is usually discarded in the strong scattering limit in favor of the distorted wave Born approximation [1] in the case of flat surfaces with (a)

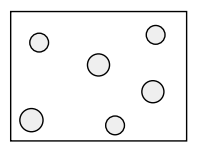

(b) Case (A)

Case (B)

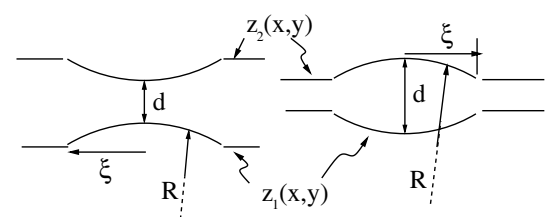

FIG. 5. (a) Top view of a random distribution of lens-shaped islands, and (b) two possible models of the lens morphologies: concave [model (A)] and convex [model (B)] lens-shaped islands. $R$ is the curvature radius, $\xi$ is the cutoff radius of the island, and $d$ is the characteristic distance between two curved surfaces.

nanoscale roughness fluctuations, as in that case a strong specularly reflected beam exists which must be taken into account. However, in the case of a curved surface no such focused specularly reflected wave exists which can interfere with the diffusely scattered beam, and thus the kinematic approximation is more likely to be valid.

We considered two possible models of the lens morphologies, $z_{1(2)}(x, y)$, as shown in Fig. 5(b). We can choose the origin of $(x, y)$ to be at the center of the lens. If the radii of curvature of the curved surfaces are much larger than their lateral size, each curved surface can be assumed to be the surface on a sphere and then approximated by

$$
\begin{aligned}
& z_{1}(x, y)= \pm\left(R-\sqrt{R^{2}-x^{2}-y^{2}}\right) \approx \pm \frac{x^{2}+y^{2}}{2 R}, \\
& z_{2}(x, y)=d \pm\left(R-\sqrt{R^{2}-x^{2}-y^{2}}\right) \approx d \pm \frac{x^{2}+y^{2}}{2 R},
\end{aligned}
$$

where \pm corresponds to concave and convex surfaces, respectively, and $d$ is the film thickness shown in Fig. 5(b). The integrals in Eq. (1) do not extend over all $(x, y)$ but are cut off at the lateral radius of the lens-shaped region. However, there will be a distribution of such sizes and such cutoffs. Thus, in keeping with the spirit of solutions to similar problems [13], we take these distributions into account by multiplying the integrands of Eq. (1) by $e^{-\left(x^{2}+y^{2}\right) / 2 \xi^{2}}$, where $\xi$ represents the average cutoff length for the lenses. The integration over the coarse out-of-plane distribution of $q_{y}$ vectors reduces the integrals to $1 \mathrm{D}$ integrals over $x$, and we finally obtain the analytic expressions

$$
I\left(q_{x}, q_{z}\right)=\frac{4 \pi \rho_{0}^{2} \xi^{2}}{q_{z}^{2}} \frac{e^{-\left(q_{x}^{2} \xi^{2} / 2\right)\left(1 / 1+\nu^{2}\right)}}{\sqrt{1+\nu^{2}}} \times\left[1-\cos \left(q_{z} d \mp \frac{q_{x}^{2} \xi^{2}}{2} \frac{\nu}{1+\nu^{2}} \pm \arctan (\nu)\right)\right]
$$

where $\nu$ is a dimensionless parameter defined by $\nu=$ $q_{z} \xi^{2} / 2 R$. The upper and lower signs in Eq. (3) represent concave [model (A)] and convex [model (B)] lens-shaped anticonformal models, respectively, as shown in Fig. 5(b). The Gaussian envelope function $\exp \left[-q_{x}^{2} \xi^{2} / 2\left(1+\nu^{2}\right)\right]$ in Eq. (3) leads to a broadening of the width along the $q_{x}$ direction by $\sqrt{1+\nu^{2}} / \xi$, which can be estimated from the broad peak in Fig. 4.

Note that, unlike models with conformal surfaces, our models with curved anticonformal surfaces, models (A) and (B), have additional $q_{x}$-dependent terms in the 
argument of the cosine function in Eq. (3). For anticonformal lens-shaped surfaces shown in Fig. 5(b), Eq. (3) yields an isointensity contour pattern in $\left(q_{x}, q_{z}\right)$ space, which is shown in the inset in Fig. 4 and which contains boomerang-shaped fringes. These give a broad single peak in the transverse scans when they pass through the maxima of the fringes at $q_{x}=0$ and a broad double peak when they pass through minima, as shown in Fig. 4. For off-specular ( $q_{x} \neq 0$ ) scans parallel to the $q_{z}$ direction, it can be seen that the fringes will go out of phase with those along $q_{x}=0$, as shown in Fig. 3. On the other hand, for models with conformal surfaces, the oscillations in the specular and off-specular scans are always in phase due to the absence of any $q_{x}$ dependence in the argument of the cosine functions. Therefore, we see that the phase between maxima positions in specular and longitudinal off-specular scans can determine unambiguously long length-scale surface morphologies between conformal and anticonformal ones.

For models (A) and (B), the condition for maxima in the oscillations can be given from Eq. (3) by

$$
q_{z} d \mp \frac{q_{x}^{2} \xi^{2}}{2} \frac{\nu}{1+\nu^{2}} \pm \arctan (\nu)=2 \pi\left(n-\frac{1}{2}\right),
$$

where $n=1,2,3, \ldots$, and upper and lower signs represent models (A) and (B), respectively. If $\nu \gg 1, \nu /\left(1+\nu^{2}\right) \approx$ $1 / \nu$ and $\arctan (\nu)$ can be approximated by $\pi / 2$, and then Eq. (4) can be approximated by

$$
q_{z}\left(d \mp \theta_{\mathrm{off}}^{2} R\right)=2 \pi\left(n-\frac{1}{2} \mp \frac{1}{4}\right) .
$$

This equation is linear in the variables $q_{z}$ and $n$, so its slope can be changed by different offset angles. This is completely consistent with the result of measurements in the inset of Fig. 3, where $q_{z}$ 's of maxima in the oscillations are plotted as a function of index $n$ for off-specular reflectivities with different offset angles. Note that their offset angles should be effectively $-0.05^{\circ}$ and $+0.15^{\circ}$ with respect to the center of the broad peak, as shown in Fig. 4, because the broad peaks are tilted from the specular ridge along the $q_{z}$ direction by a constant angle, i.e., $-0.05^{\circ}$, as discussed earlier. From the slopes in the inset of Fig. 3 and Eq. (5), our freestanding film is readily found to have convex lens-shaped anticonformal morphology, and the unknown parameters, i.e., the maximum distance $d$ between two curves and the curvature radius $R$, can be estimated.

To refine the parameters, the longitudinal and transverse off-specular reflectivity data were fitted simultaneously using the full expression of Eq. (3) for curved surfaces and the asymptotic power-law expression in Ref. [12] for capillary wave fluctuations at small wavelength scales. The solid lines in Fig. 3 and thick solid lines in Fig. 4 represent the results of the fitting. The thin solid lines and dashed lines in Fig. 4 represent the contributions from curved surfaces and capillary wave fluctuations, respectively. The thin solid lines in Figs. 4(a) and 4(b) show clearly that the positions of maximum intensities along the $q_{x}$ direction change dramatically between the scattering intensities from the crest [4(a)] and the valley [4(b)] of the specular reflectivity curve, as indicated by arrows in Fig. 3. The best fit yields the maximum distance $d$ between two facing surfaces of $317.7( \pm 0.5) \AA$, which is slightly larger than the average film thickness estimated from the specular reflectivity. The curvature radius $R$ and the island cutoff length $\xi$ were estimated to be $2.7( \pm 0.2) \times 10^{6} \AA$ and $7.0( \pm 1.0) \times 10^{3} \AA$, respectively, which yields the parameter $\nu$ of $9.1 \AA \times q_{z}$. The value of $\nu$ was initially estimated from the slopes of maxima in the oscillations and refined by fitting of the detailed spectra in Figs. 3 and 4.

In summary, we demonstrated using x-ray specular and off-specular reflectivity measurements that a freestanding polymer thin film has anticonformally curved lens-shaped islands at long length scales and large separations. We have proposed a geometric description for the height morphologies of two conformal or anticonformal surfaces and revealed from the analytic calculation of the reflected intensities that the phase between the maxima positions in the longitudinal off-specular scans can unambiguously distinguish between conformal and anticonformal morphologies at the two opposing surfaces.

The authors thank Y. Chu at Argonne for valuable discussions. Work at Argonne is supported by the U.S. DOE, Office of Basic Energy Sciences, Contract No. W-31109-ENG-38. S. K. S. is supported by the U.S. DOE, BESDMS, under Contract No. W-7405-ENG-36.

[1] S. K. Sinha, E. B. Sirota, S. Garoff, and H. B. Stanley, Phys. Rev. B 38, 2297 (1988).

[2] Y. H. Phang, D. E. Savage, R. Kariotis, and M. G. Lagally, J. Appl. Phys. 74, 3181 (1993).

[3] V. Holý and T. Baumbach, Phys. Rev. B 49, 10668 (1994).

[4] S. K. Sinha, J. Phys. III 4, 1543 (1994).

[5] G. Vignaud, A. Gibaud, J. Wang, S. K. Sinha, J. Daillant, G. Grübel, and Y. Gallot, J. Phys. Condens. Matter 9, L125 (1997).

[6] T. Salditt, T. H. Metzger, and J. Peisl, Phys. Rev. Lett. 73, 2228 (1994).

[7] R. Paniago, H. Homma, P. C. Chow, S. C. Moss, Z. Barnea, S.S. P. Parkin, and D. Cookson, Phys. Rev. B52, R17 052 (1995).

[8] A. Gibaud, N. Cowlam, G. Vignaud, and T. Richardson, Phys. Rev. Lett. 74, 3205 (1995).

[9] R. E. Geer, R. Shashidhar, A. F. Thibodeaux, and R. S. Duran, Phys. Rev. Lett. 71, 1391 (1993).

[10] J. D. Shindler, E. A. L. Mol, A. Shalaginov, and W. H. de Jeu, Phys. Rev. Lett. 74, 722 (1995).

[11] E. A. L. Mol, G. C. L. Wong, J. M. Petit, F. Rieutord, and W. H. de Jeu, Phys. Rev. Lett. 79, 3439 (1997).

[12] K. Shin, Y. Pu, M. H. Rafailovich, J. Sokolov, O. H. Seeck, S. K. Sinha, M. Tolan, and R. Kolb, Macromolecules 34, 5620 (2001).

[13] P. Dutta and S. K. Sinha, Phys. Rev. Lett. 47, 50 (1981). 